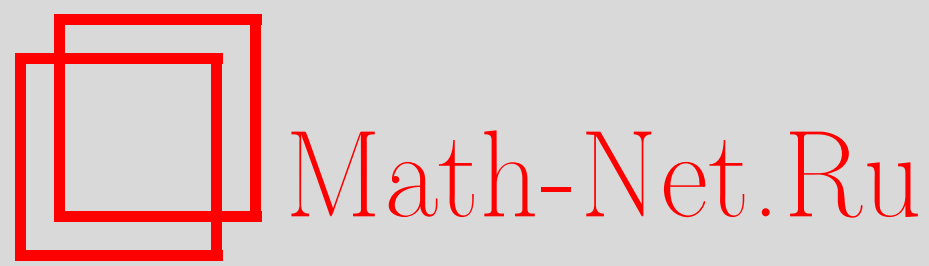

В. В. Волчков, Экстремальные варианты проблемы Помпейю, Матем. заметки, 1996, том 59, выпуск $5,671-680$

DOI: https://doi.org/10.4213/mzm1761

Использование Общероссийского математического портала MathNet.Ru подразумевает, что вы прочитали и согласны с пользовательским соглашением

http://www . mathnet.ru/rus/agreement

Параметры загрузки:

IP : 54.89 .56 .158

26 апреля 2023 г., 14:06:53

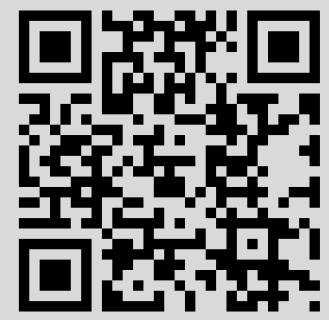


тОм 59 выПУСК 5 мАй 1996

\section{ЭКСТРЕМАЛЬНЫЕ ВАРИАНТЫ ПРОБЛЕМЫ ПОМПЕЙЮ}

\section{В.В. Волчков}

1. Введение. Пусть $M(n)$ - группа изометрий вещественного евклидова пространства $\mathbb{R}^{n}, n \geqslant 2$. Компактное множество $K \subset \mathbb{R}^{n}$ называется множеством Помпейю, если всякая локально суммируемая функция $f$ $\left(f \in L_{\mathrm{loc}}\left(\mathbb{R}^{n}\right)\right)$, для которой

$$
\int_{\sigma K} f(u) d u=0 \quad \text { для всех } \sigma \in M(n),
$$

равна нулю почти всюду. Классическая проблема Помпейю об описании таких $K$ изучалась многими авторами (см. обзор [1] с обширной библиографией).

Большой интерес представляют “локальные” варианты проблемы Помпейю: например, когда $f$ задана в шаре $B \subset \mathbb{R}^{n}$ и (1) вьполнено при всех $\sigma K \subset B$. В этом случае для широкого класса множеств $K$ следует, что $f=0$ в $B$, если только размеры $B$ достаточно велики по сравнению с $K$ (см. [2]). В связи с этим возникает задача о нахождении минимального радиуса $r=r(K)$ шара $B$ с этим свойством. До настояшего времени точное значение $r(K)$ не было известно ни при каком $K$. Вместе с тем известен ряд результатов (см. [2], [3]), содержащих оценки $r(K)$ для многих $K$.

В данной работе получено полное решение поставленной задачи в следующих случаях:

a) $K=[0,1]^{n}$ - единичный куб;

б) $K$ - единичный полушар, т.е. $K=\left\{x=\left(x_{1}, \ldots, x_{n}\right) \in \mathbb{R}^{n}: x_{1} \geqslant 0\right.$, $|x| \leqslant 1\}$, где $|\cdot|$ - евклидова норма в $\mathbb{R}^{n}$.

2. Основные результаты. Пусть $B_{r}=\left\{x \in \mathbb{R}^{n}:|x|<r\right\}$.

ТЕОрема 1. Пусть $f \in L_{\mathrm{loc}}\left(B_{r}\right)$ и для любого единичного куба $K \subset B_{r}$

$$
\int_{K} f(u) d u=0
$$


Тогда справедливы следующие утверждения.

1) Eсли $r \geqslant \frac{1}{2} \sqrt{n+3}$, mo $f=0$.

2) При $r<\frac{1}{2} \sqrt{n+3}$ существуют ненулевые функции класса $C^{\infty}\left(B_{r}\right)$ с условием (2).

Отметим, что при $r>\sqrt{n}$ первое утверждение теоремы 1 было получено Беренстейном и Гэем [2], [3]. При $r>\frac{1}{2} \sqrt{n+3}$ этот результат получил автор [4].

ТЕОрема 2. Пусть $f \in L_{\mathrm{loc}}\left(B_{r}\right)$ и для любого единичного полуиара $P \subset B_{r}$

$$
\int_{P} f(u) d u=0 .
$$

Тогда выполнены следуюшие утверждения.

1) $E$ сли $r \geqslant \sqrt{5} / 2$, mo $f=0$.

2) При $r<\sqrt{5} / 2$ существуют ненулевые функции класса $C^{\infty}\left(B_{r}\right)$ с условием (3).

При $r>2$ первое утверждение теоремы 2 следует из общей оценки для $r(K)$ (см. [2], [3]). Отметим также [5], где подобная задача изучалась на $\mathbb{R}^{2}$.

3. Вспомогательные утверждения. Здесь мы введем необходимые обозначения и докажем некоторые утверждения, представляюшие самостоятельный интерес.

Пусть $\rho, \sigma$ - полярные координаты в $\mathbb{R}^{n}$ (для $x \in \mathbb{R}^{n} \rho=|x|$, а если $x \neq 0$, то $\sigma=x / \rho), \Omega=\left\{x \in \mathbb{R}^{n}:|x|=1\right\}, \mathrm{SO}(n)$ - групша вращений с нормированной мерой Хаара $d g$. Обозначим $T(g)$ квазирегулярное представление (для $f \in L^{2}(\Omega)(T(g) f)(\sigma)=f\left(g^{-1} \sigma\right)$, где $\sigma \in \Omega$, $g \in \mathrm{SO}(n))$. Как известно (см. [6, с. 442]), $T(g)$ является прямой суммой попарно неэквивалентных неприводимых унитарных представлений $T^{k}(g)$, действующих на пространствах $H_{k}$ однородных гармонических полиномов степени $k$. Пусть $\left\{Y_{l}^{(k)}\right\}\left(1 \leqslant l \leqslant a_{k}\right)$ - ортонормированньй базис в $H_{k}$ (пространство $H_{k}$ рассматривается как подпространство $L^{2}(\Omega)$ ), $\left\{t_{l p}^{k}\right\}\left(1 \leqslant l, p \leqslant a_{k}\right)$ - матрица представления $T^{k}(g)$ :

$$
\left(T^{k}(g) Y_{l}^{(k)}\right)(\sigma)=Y_{l}^{(k)}\left(g^{-1} \sigma\right)=\sum_{p=1}^{a_{k}} t_{l p}^{k}(g) Y_{p}^{(k)}(\sigma) .
$$

Всякой функции $f \in L_{\mathrm{loc}}\left(B_{r}\right)$ соответствует ряд Фурье

$$
f(x) \sim \sum_{k=0}^{\infty} \sum_{l=1}^{a_{k}} f_{k l}(\rho) Y_{l}^{(k)}(\sigma), \quad 0<\rho<r,
$$


где

$$
f_{k l}(\rho)=\int_{\Omega} f(\rho \sigma) \overline{Y_{l}^{(k)}(\sigma)} d \sigma .
$$

Для последовательности $f_{k l}$, определенной формулой $(6)$, имеется другое представление (см. [7]):

$$
f_{k l}(\rho) Y_{p}^{(k)}(\sigma)=a_{k} \int_{\mathrm{SO}(n)} f\left(\rho g^{-1} \sigma\right) \overline{t_{l p}^{k}(g)} d g
$$

Обозначим $\mathscr{D}\left(B_{r}\right)$ - множество функций $f \in C^{\infty}\left(B_{r}\right)$ с компактным носителем, $\mathscr{D}_{0}\left(B_{r}\right)$ - множество радиальных (зависящих только от $\rho$ ) функций из $\mathscr{D}\left(B_{r}\right)$. Для $x=\left(x_{1}, \ldots, x_{n}\right) \in \mathbb{R}^{n}$ положим $[x]=x_{1}$. Пусть еще $\chi_{r}=\chi_{r}(x)$ - характеристическая функция (индикатор) шара $B_{r}, \Delta$ - оператор Лапласа, $\varphi * \psi-$ свертка функций $\varphi$ и $\psi$. Символами $c, c_{1}, c_{2}, \ldots$ обозначаются комплексные постоянные, которые в разных случаях, вообще говоря, разные.

Получим теперь общее решение одного функционального уравнения, впервые рассмотренного в [5].

Tеорема 3. Пусть $0<\delta<1, K_{\delta}=\left\{x \in \mathbb{R}^{n}: 1-\delta<|x|<1+\delta\right\}$, $f \in C\left(K_{\delta}\right)$ u nрu всех $u, v \in \Omega, w \in B_{\delta}$

$$
f(w+u)+f(w-u)=f(w+v)+f(w-v) .
$$

To2 $\partial a$

$$
f(x)=c_{1}|x|^{2}+c_{2}(x, y)+c_{3}
$$

где у $\in \Omega,(\cdot, \cdot)$ - скалярное произведение в $\mathbb{R}^{n}$. Обратно, всякая функиия вида (9) удовлетворяет (8).

ДокАЗАТЕЛЬство. Пусть $S_{n}$-множество функций $f \in C^{\infty}\left(K_{\delta}\right)$, удовлетворяющих (8). Если $n=2$, то для $f=f(x, y) \in S_{2}$ при $\alpha \in \mathbb{R}^{1},|t|<\delta$ имеем

$$
f(t+1,0)+f(t-1,0)=f(t+\cos \alpha, \sin \alpha)+f(t-\cos \alpha,-\sin \alpha) .
$$

Дифференцируя по $\alpha$ и полагая $\alpha=0$, получим

$$
\frac{\partial f}{\partial y}(1+t, 0)=\frac{\partial f}{\partial y}(t-1,0)
$$

Далее точка $(x, y) \in \mathbb{R}^{2}$ отождествляется с комплексным числом $z=x+i y$. Изучим функции вида $f_{k}=u_{k}(|z|) z^{k} \in S_{2}, k=0,1, \ldots$. 
При $k=1$ из (10) следует, что

$$
u_{1}^{\prime}(1+t)=-u_{1}^{\prime}(1-t) .
$$

Далее, вместе с $f$ и все частные производные от $f$ очевидно принадлежат $S_{2}$. Применяя (11) к функции $\partial^{2} f / \partial y^{2}$ получим

$$
\frac{u_{1}^{\prime}(1+t)}{1+t}=\frac{u_{1}^{\prime}(1-t)}{1-t} \quad \text { при }|t|<\delta .
$$

Отсюда и из (11) следует, что $f_{1}=c z$.

Докажем теперь индукцией по $k=2,3, \ldots$, что $f_{k}=0$. При $k=2$ имеем $\partial f_{2} / \partial z=z\left(u_{2}^{\prime}(|z|) /|z|+2 u(|z|) /|z|^{2}\right) \in S_{2}$ и по доказанному выше $f_{2}=z\left(c_{1}|z|^{-3}+c_{2}|z|\right)$. Легко проверить, что такие функции принадлежат $S_{2}$ только при $c_{1}=c_{2}=0$. Предположим, что при некотором $k \geqslant 2$ всякая $f_{k} \in S_{2}$ равна нулю. Пусть $f_{k+1} \in S_{2}$, тогда $\partial f_{k+1} / \partial z=$ $z^{k}\left(u_{k+1}^{\prime}(|z|)|z|^{-k}+(k+1) u_{k+1}(|z|)|z|^{-k-1}\right.$ и по предположению индукции $f_{k+1}=c|z|^{-2 k-2} z^{k+1}$. Поскольку $f_{k+1} \in S_{2}, c=0$ и $f_{k+1}=0$.

Пусть $k=0, f_{0} \in S_{2}$. Тогда $\partial f_{0} / \partial z=u_{0}^{\prime}(|z|) z /|z| \in S_{2}$ и по доказанному вьше $f_{0}=c_{1}|z|^{2}+c_{2}$.

Рассмотрим теперь произвольную функцию $f \in S_{n}, n \geqslant 2$. Поскольку $\forall g \in \mathrm{SO}(n) f(g x) \in S_{n}$, из (7) следует, что $f_{k l}(\rho) Y_{p}^{(k)}(\sigma) \in S_{n}$ при всех $1 \leqslant l, p \leqslant a_{k}, k=0,1, \ldots$. Тогда для любого $Y \in H_{k} \quad f_{k l}(\rho) Y(\sigma) \in S_{n}$. Положим $Y(\sigma)=\left(\sigma_{1}+i \sigma_{2}\right)^{k}$ и рассмотрим сужение $f_{k l}(\rho) Y(\sigma)$ на плоскость $\left\{x \in \mathbb{R}^{n}: x_{i}=0\right.$ при $\left.i>2\right\}$. Тогда $f_{k l}(|z|) z^{k}|z|^{-k} \in S_{2}$ и по доказанному выше $f_{k l}=0$ при $k \geqslant 2, f_{1 l}=c \rho, f_{0 l}=c_{1} \rho^{2}+c_{2}$. Отсюда следует утверждение теоремы 3 для $f \in S_{n}$. Пусть теперь $f \in C\left(K_{\delta}\right)$ и удовлетворяет (8). Тогда $f$ можно приблизить равномерно на компактах в $K_{\delta}$ свертками вида $f * \varphi_{\varepsilon} \in C^{\infty}$, где $\varphi_{\varepsilon} \in \mathscr{D}\left(B_{\varepsilon}\right)$ (см. [8, c. 17]). Очевидно, при $\varepsilon<\delta$ такие свертки удовлетворяют (8) при всех $u, v \in \Omega, w \in B_{\delta-\varepsilon}$. По доказанному вьше такие свертки имеют вид (9) и в силу замкнутости конечномерного линейного многообразия в банаховом пространстве при $\varepsilon \rightarrow 0$ получаем, что $f$ также имеет вид (9).

Обратное утверждение теоремы 3 тривиально.

Докажем теперь теорему единственности для функций с нулевыми шаровыми средними.

Tеорема 4. Пусть $r>1,0<\alpha<\beta \leqslant r-1, f \in L_{\mathrm{loc}}\left(B_{r}\right) u$ имеет нулевые интегралы по всем замкнутым единичным шарам, содерэсащимся в $B_{r}$. Тогда справедливы следующие утверждения.

1) Если $f=0$ в кольие $1-\alpha<|x|<1+\beta$, то $f=0$ и в кольие $1-\beta<|x|<1+\beta$. 
2) Если $f=0$ в кольце $1-\beta<|x|<1+\alpha$, то $f=0$ и в кольие $1-\beta<|x|<1+\beta$.

Для доказательства потребуется

Лемма 1. Пусть $u \in \mathscr{D}_{0}\left(B_{r}\right)$. Тогда уравнение

$$
\int_{\mathrm{SO}(n)} h([g x]) d g=u(x)
$$

имеет единственное решение $h$ в классе четных непрерывных на $[-r, r]$ функиий.

ДоказАТЕЛЬСтво. Уравнение (12) можно переписать в виде $u(x)=$ $\int_{\Omega} h([\rho \sigma]) d \sigma$ (см. $[8$, c. 46]). При четных $h$ последнее уравнение сводится к уравнению Абеля [9, с. 126], откуда следует утверждение леммы 1.

Оба утверждения теоремы 4 достаточно доказать для радиальной функции $f \in C^{\infty}\left(B_{r}\right)$ (см. конец доказательства теоремы 3 , а также [10, доказательство леммы 2]). Поэтому далее считаем $f(x)=F(\rho)$, где $F \in C^{\infty}[0, r)$. Положим $F=0$ на $[r, \infty)$.

Докажем первое утверждение. Пусть $0<\varepsilon<\alpha / 2, \psi_{\varepsilon} \in \mathscr{D}_{0}\left(B_{\varepsilon}\right)$, $\psi_{\varepsilon} \geqslant 0, \int_{B_{\varepsilon}} \psi_{\varepsilon}(x) d x=1, \varphi=\psi_{\varepsilon} * \chi_{1}$. Тогда $\varphi \in \mathscr{D}_{0}\left(B_{1+\varepsilon}\right)$ и при $|y|<r-1-\varepsilon$

$$
\int_{B_{r}} f(x) \varphi(y-x) d x=0 .
$$

Пусть $h=h([x])-$ решение (12) при $u=\varphi$. Из определения $\varphi$ и свойств $\psi_{\varepsilon}$ имеем $\varphi=1$ в $B_{1-\varepsilon}, 0 \leqslant \varphi<1$ вне $B_{1-\varepsilon}$. По лемме 1 отсюда $h=1$ в $B_{1-\varepsilon}$ и при любом $\gamma>0$

$$
\operatorname{supp}(h-1) \cap B_{1-\varepsilon+\gamma} \neq \varnothing .
$$

Пусть $v([y])=(h * F)(y)$, тогда $\forall g \in \mathrm{SO}(n) v([g y])=\int_{\mathbb{R}^{n}} h([g x]) F(|y-x|) d x$. Интегрируя это равенство по $\mathrm{SO}(n)$, из (13), определения $h$ и леммы 1 при $u=0$ получим $v([y])=0$ в $B_{r-1-\varepsilon}$. Тогда

$$
((h-1) * F)(y)=-\int_{\mathbb{R}^{n}} F(|x|) d x, \quad y \in B_{r-1-\varepsilon} .
$$

Пусть $\Phi=F$ на $[0,1+\beta), \Phi=0$ на $[1+\beta, \infty), \eta=\Delta(\Phi(|x|))$. Тогда из $(15)$ при $|y|<\beta-\varepsilon$ имеем

$$
\int_{\mathbb{R}^{n}}(h([x])-1) \eta(|y-x|) d x=0 .
$$


Далее проверяются известные рассуждения [10, доказательство леммы 2]: уравнение (16) приводится к уравнению Вольтерра первого рода, затем из теоремы Титчмарша $[11$, с. 608$]$, теоремы о носителе [9, с. 125] и (14) получаем $F=0$ на $(1-\beta+\varepsilon, 1+\beta)$. В силу произвольности $\varepsilon$ отсюда следует первое утверждение теоремы 4.

Для доказательства второго утверждения этой теоремы положим $H(x)=f(x)$ в $B_{1+\alpha}, H(x)=0$ при $1+\alpha \leqslant|x| \leqslant 1+\beta$. Тогда функции $f, H, f-H$ удовлетворяют (13) при $|y|<\beta-\varepsilon$ (поскольку $\varphi=1$ в $B_{1-\varepsilon}, \varphi=0$ вне $\left.B_{1+\varepsilon}\right)$. Так как $f-H=0$ в $B_{1+\alpha}$ из [10] получаем, что $f=H$ в $B_{1+\beta-\varepsilon}$. Из произвольности $\varepsilon$ и определения $H$ получаем второе утверждение теоремы 4 .

4. Доказательство теоремы 1. Пусть $r>\sqrt{n} / 2$. Обозначим $\mathscr{K}_{r}-$ класс функций $f \in C^{\infty}\left(B_{r}\right)$, имеющих нулевые интегралы по всем единичным кубам $K \subset B_{r}$. Нам потребуются следуюшие простые свойства $\mathscr{K}_{r}$, которые уже использовались в предшествующих работах.

Лемма 2. Пусть $f \in \mathscr{K}_{r}$. Тогда справедливы следующие утвержсдения.

1) $f(g x) \in \mathscr{K}_{r}$ для любого $g \in \mathrm{SO}(n)$.

2) Каждое слагаемое ряда (5), доопределенное по непрерывности в точке $x=0$, принадлежит $\mathscr{K}_{r}$.

3) Все частные производные $f$ принадлежат $\mathscr{K}_{r}$.

4) $\int_{K_{n-1}} f\left(x_{1}, \ldots, x_{n-1},-\frac{1}{2}\right) d \mu=\int_{K_{n-1}} f\left(x_{1}, \ldots, x_{n-1}, \frac{1}{2}\right) d \mu$, где $d \mu=d x_{1} \cdots d x_{n-1}, K_{n-1}=[-1 / 2,1 / 2]^{n-1}$.

5) $\int_{K_{n-2}} f\left(x_{1}, \ldots, x_{n-1},-\frac{1}{2},-\frac{1}{2}\right) d \nu+\int_{K_{n-2}} f\left(x_{1}, \ldots, x_{n-1}, \frac{1}{2}, \frac{1}{2}\right) d \nu$ $=\int_{K_{n-2}} f\left(x_{1}, \ldots, x_{n-2},-\frac{1}{2}, \frac{1}{2}\right) d \nu+\int_{K_{n-2}} f\left(x_{1}, \ldots, x_{n-2}, \frac{1}{2},-\frac{1}{2}\right) d \nu$, əде $n>2, d \nu=d x_{1} \cdots d x_{n-2}, K_{n-2}=[-1 / 2,1 / 2]^{n-2}$.

6) Пусть $r<\frac{1}{2} \sqrt{n+3}, B$ - пересечение всех единичных кубов $K \subset B_{r}$. Тогда, если $f$-многочлен в $B_{r} \backslash B$, то $f=c$ в $B_{r} \backslash B$.

7) Пусть $f(x)=F(|x|) P(x)$, где $P$ - однородный гармонический многочлен степени $k \geqslant 1$. Тогда для любого однородного гармонического многочлена $Q$ степени $k-1$ функиия $\left(|x| F^{\prime}(|x|)+(n+2 k-2) F(|x|)\right) Q(x)$ принадлежит $\mathscr{K}_{r}$.

ДоКАЗАТЕЛЬСтво. Первое утверждение леммы 2 очевидно. Второе утверждение сразу следует из первого и формулы (7). Для доказательства третьего утверждения достаточно продифференцировать свертку $f$ с 
индикатором единичного куба. Утверждения 4, 5 легко получить, интегрируя по кубу $[-1 / 2,1 / 2]^{n}$ функции $\partial f / \partial x_{n} \in \mathscr{K}_{r}, \partial^{2} f / \partial x_{n-1} \partial x_{n} \in \mathscr{K}_{r}$. Утверждение 6 очевидно, если $f$-полином не выше первой степени в $B_{r} \backslash B$. В обшем случае нужно сначала понизить степень $f$ дифференцированием, а затем воспользоваться утверждением 3 . Доказательство утверждения 7 содержится в работе [7].

Лемма 3. Пусть $r=\frac{1}{2} \sqrt{n+2}, f \in \mathscr{K}_{r}$. Тогда $f=c$ в кольце $1-\sqrt{3} / 2<|x|<r$.

ДОКАЗАТЕЛЬСТВО. Из утверждений $1,2,6,7$ леммы 2 заключаем (см. $[10$, доказательство леммы 2]), что лемму 3 достаточно доказать для радиальных функций $f \in \mathscr{K}_{r}$. Пусть $n=2$. Из условия следует [4], что $\Delta^{2} f=0$ при $1 / 2<|x|<1$ и, что интеграл $\Delta^{2} f$ по любой хорде круга $|x| \leqslant 1 / 2$, не пересекающей круг $|x| \leqslant 1-\sqrt{3} / 2$, равен нулю. По теореме о носителе [9, с.125] $\Delta^{2} f=0$ при $1-\sqrt{3} / 2<|x|<1$. Отсюда $\Delta f=c \ln \rho+c_{1}$ в этом кольце. Так как $\Delta f \in \mathscr{K}_{r}, c=0$ и $f=\frac{1}{4} c_{1} \rho^{2}+c_{2}$ при $1-\sqrt{3} / 2<|x|<1$. Поскольку $f \in \mathscr{K}_{r}, c_{1}=0$ и мы получаем $f=c_{2}$ при $1-\sqrt{3} / 2<|x|<1$.

Предположим, что утверждение леммы 3 верно для любой размерности от 2 до $n-1$ и докажем его для размерности $n$. Пусть

$$
f=F(|x|), \quad \Phi=\frac{\partial f}{\partial x_{n}}=F^{\prime}(|x|) \frac{x_{n}}{|x|} \in \mathscr{K}_{r}
$$

В этом случае интегралы от $\Phi$ по противоположным $(n-1)$-мерным граням любого единичного куба $K \subset B_{r}$ равны (см. утверждение 4 леммы 2) и в силу нечетности $\Phi$ по $x_{n}$ получаем, что функция

$$
\frac{1}{2} F^{\prime}\left(\sqrt{\frac{1}{4}+x_{1}^{2}+\cdots+x_{n-1}^{2}}\right)\left(\frac{1}{4}+x_{1}^{2}+\cdots+x_{n-1}^{2}\right)^{-1 / 2}
$$

удовлетворяет условию леммы в шаре $x_{1}^{2}+\cdots+x_{n-1}^{2}<\frac{1}{4}(n+1)$. По предположению индукции отсюда следует, что

$$
F^{\prime}=c|x| \quad \text { при }\left(\frac{1}{4}+\left(1-\frac{\sqrt{3}}{2}\right)^{2}\right)^{1 / 2}<|x|<\frac{\sqrt{n+2}}{2} \text {. }
$$

Следовательно, при таких $x \Delta^{2} f=0$. Так как $\Delta^{2} f \in \mathscr{K}_{r}$, сдвигая куб $[-1 / 2,1 / 2]^{n}$ на вектор вида $(0, \ldots, 0, t, t) \in \mathbb{R}^{n}$ до касания с границей $B_{r}$, из утверждения 5 леммы 2 и радиальности $\Delta^{2} f$ получаем, что 
интеграл от $\Delta^{2} f$ по пересечению $B_{r}$ с любой $(n-2)$-мерной гиперплоскостью, не пересекающей шар $B_{\sqrt{2}-1}$, равен нулю. По теореме о носителе $\left[9\right.$, c.125] $\Delta^{2} f=0$ вне $B_{\sqrt{2}-1}$. Далее, сдвигая куб $[-1 / 2,1 / 2]^{n}$ на вектор вида $(0, \ldots, 0, t) \in \mathbb{R}^{n}$ до касания с гранищей $B_{r}$, из утверждения 4 леммы 2 получаем, что интеграл от $\Delta^{2} f$ по пересечению $B_{\sqrt{2}-1}$ с любой $(n-1)$-мерной гиперплоскостью, не пересекающей шар $B_{1-\sqrt{3} / 2}$, равен нулю. По теореме о носителе $[9, \mathrm{c.125}] \Delta^{2} f=0$ вне $B_{1-\sqrt{3} / 2}$. Отсюда и из (17) следует, что $\Phi=c x_{n}$ вне $B_{1-\sqrt{3} / 2}$. Из утверждения 6 леммы 2 получаем $c=0$, откуда следует утверждение леммы 3 .

Перейдем к доказательству теоремы 1 . Пусть $r \geqslant \frac{1}{2} \sqrt{n+3}, \varepsilon>0$ достаточно мало, $\psi_{\varepsilon}$ - функция, использованная в доказательстве теоремы 4. Тогда $f * \psi_{\varepsilon} \in \mathscr{K}_{r-\varepsilon}$. Представляя $B_{r-\varepsilon}$ в виде объединения шаров радиуса $\frac{1}{2} \sqrt{n+2}$ из леммы 3 имеем $f * \psi_{\varepsilon}=c$ при $1 / 2 \leqslant|x|<r-\varepsilon$. Тогда $f_{k}=\partial\left(f * \psi_{\varepsilon}\right) / \partial x_{k} \in \mathscr{K}_{r-\varepsilon}, 1 \leqslant k \leqslant n$, и равна нулю вне $B_{1 / 2}$. Сдвигая куб $[-1 / 2,1 / 2]^{n}$ на вектор вида $(0, \ldots, 0, t) \in \mathbb{R}^{n}$ до касания с границей $B_{r-\varepsilon}$, из утверждений 2,4 леммы 2 получаем, что интеграл от $f_{k}$ по пересечению $B_{r-\varepsilon}$ с любой $(n-1)$-мерной гиперплоскостью, не пересекающей шар $|x|<1-\sqrt{(r-\varepsilon)^{2}-(n-1) / 4}$, равен нулю. По теоремео носителе [9, c. 125] $f * \psi_{\varepsilon}=c$ вне этого шара. В силу произвольности $\varepsilon>0$ отсюда следует, что $f-$ константа в $B_{r}$ и тогда $f=0$.

Доказательство второго утверждения теоремы 1 содержится в [4].

5. Доказательство теоремы 2. Пусть $r>1$. Обозначим $P_{r}-$ класс функций $f \in C^{\infty}\left(B_{r}\right)$, имеющих нулевые интегралы по всем единичным полушарам $P \subset B_{r}$. Легко видеть, что если $f \in P_{r}$, то выполнены утверждения 1-3 леммы 2 с заменой $\mathscr{K}_{r}$ на $P_{r}$.

Лемма 4. Пусть $f \in P_{r} u f=0$ nрu $|x|>2-r$. Тогда

$$
\int_{B} f\left(x_{1}, \ldots, x_{n-1}, 0\right) d x_{1} \cdots d x_{n-1}=0,
$$

əде

$$
B=\left\{x \in \mathbb{R}^{n-1}: x_{1}^{2}+\cdots+x_{n-1}^{2} \leqslant 1\right\} .
$$

ДоКАЗАТЕЛЬСТВо. Пусть $\delta>0, P^{1}=\left\{x \in \mathbb{R}^{n}:|x| \leqslant 1,0 \leqslant x_{n}\right.$ $\left.\leqslant \delta x_{1}\right\}, P^{2}=\left\{x \in \mathbb{R}^{n}:|x| \leqslant 1, x_{1} \delta \leqslant x_{n} \leqslant 0\right\}$. Из условия следует, что $\partial f / \partial x_{1} \in P_{r}$ и $\int_{P^{1}} f d x=\int_{P^{2}} f d x$. При $\delta \rightarrow 0$ из последнего равенства имеем

$$
\int_{B} \frac{\partial f}{\partial x_{1}}\left(x_{1}, \ldots, x_{n-1}, 0\right) x_{1} d x_{1} \cdots d x_{n-1}=0 .
$$

Интегрируя по частям, получим утверждение леммы 4 . 
Лемма 5. Пусть $f \in P_{r}$. Тогда $f$ удовлетворяет (8) при всех $u, v \in \Omega, w \in B_{r-1}$.

ДоКАЗАТЕЛЬСТво. Достаточно получить (8) при $w=0$ (общий случай получается сдвигом). Пусть $P=\left\{x \in \mathbb{R}^{n}:|x| \leqslant 1, x_{n} \geqslant 0\right\}$, $S=\left\{x \in \mathbb{R}^{n}:|x|=1, x_{n} \geqslant 0\right\}$. По формуле Остроградского при $j \neq n$ имеем $\int_{S} f\left(g^{-1} \sigma\right) \sigma_{j} d \sigma=\int_{P}\left(\partial f\left(g^{-1} x\right) / \partial x_{j}\right) d x=0$ при всех $g \in \operatorname{SO}(n)$, поскольку $f \in P_{r}$. Умножая на $\overline{t_{l p}^{k}(g)}$ и интегрируя на $\mathrm{SO}(n)$, из $(7)$ получаем $f_{k l}(1) \int_{S} \sigma_{j} Y(\sigma) d \sigma=0$ при $Y=Y_{p}^{(k)}, 1 \leqslant p \leqslant a_{k}$, а значит, при всех $Y \in H_{k}$. Если $k$ четно, последний интеграл не равен нулю при $Y(\sigma)=\left(\sigma_{n-1}+i \sigma_{n}\right)^{k}$ (его легко вычислить в полярных координатах), откуда $f_{k l}(1)=0$. Тогда все слагаемые ряда (5) для функции $f(x)+f(-x)$ при $k \geqslant 1$ равны нулю, откуда следует утверждение леммы 5 .

\section{Лемма 6. Пусть $f \in P_{r}$. Тогда $f=c n p u|x|>1-\sqrt{r^{2}-1}$.}

ДоКАЗАТЕЛЬСТвО. Из условия, леммы 5 и теоремы 3 следует, что $f-$ полином при $|x|>2-r$. Поскольку $f$ имеет нулевые интегралы по всем единичньм шарам, лежащим в $B_{r}$, получаем (см. доказательство утверждения 6 леммы 2) $f=c$ вне $B_{2-r}$. Функция $f_{k}=\partial f / \partial x_{k}(1 \leqslant k \leqslant n)$ удовлетворяет условиям леммы 4. Положим $f_{k}=0$ при $r \leqslant|x| \leqslant R$, где $R=1+\sqrt{r^{2}-1}$. Отметим, что $B_{R}$ представляет собой наименьший шар, который вместе с любым единичньм полушаром $P \subset B_{r}$ содержит единичньй шар, содержаший $P$. Тогда из леммы 4 имеем, что $f_{k}$ имеет нулевые интегралы по всем единичньм шарам из $B_{R}$ (для шаров с центрами на оси $O x_{n}$ это очевидно, и остается учесть, что $f_{k}(g x) \in P_{r}$ при $g \in \mathrm{SO}(n))$. Теперь из теоремы 4 следует утверждение леммы 6 .

Докажем первое утверждение теоремы 2 . Пусть $0<\varepsilon<\sqrt{5} / 2-1, \psi_{\varepsilon}-$ функция из доказательства теоремы 4 . Тогда свертка $f * \psi_{\varepsilon}$ удовлетворяет условиям леммы 6 при $r=\sqrt{5} / 2-\varepsilon$. Из леммы 6 и произвольности $\varepsilon>0$ следует $f=c$ вне $B_{1 / 2}$. Из условия следует, что функция $f-c$ имеет равные интегралы по всем единичньм полушарам $P \subset B_{\sqrt{5} / 2}$. Отсюда получаем, что интеграл от $f-c$ по пересечению $B_{1 / 2}$ с любой полосой вида $g Q$, где $g \in \mathrm{SO}(n), Q=\left\{x \in \mathbb{R}^{n}: h_{1} \leqslant x_{n} \leqslant h_{2}\right\}$, равен нулю. Из инъективности преобразования Радона [9, с. 142] получаем $f=c$ в $B_{1 / 2}$. Итак, $f=c$ в $B_{\sqrt{5} / 2}$, откуда $f=0$.

Для доказательства второго утверждения теоремы 2 при $1<r<\sqrt{5} / 2$ рассмотрим линейно независимые радиальные функции $g_{1}, g_{2} \in C^{\infty}\left(\mathbb{R}^{n}\right)$, равные нулю при $|x| \leqslant \sqrt{r^{2}-1}$ и при $|x| \geqslant 1-\sqrt{r^{2}-1}$. Пусть $g_{1}$ и $g_{2}$ - преобразования Радона функций $f_{1}, f_{2}$ (см. [9, с. 120]). По теореме о носителе [9, с. 125] $f_{1}=f_{2}=0$ при $|x| \geqslant 1-\sqrt{r^{2}-1}$. Поскольку $r-1<\sqrt{r^{2}-1}$, всякий единичный полушар $P \subset B_{r}$ содержит сегмент 
$H$ шара $B_{R}, R=1-\sqrt{r^{2}-1}$ с высотой $2 R-1$, причем основание $H$ параллельно основанию $P$. Пусть $f=\alpha f_{1}+\beta f_{2}$, где $f \neq 0, \int_{H} f(x) d x=0$. $\Phi$ ункция $f$ удовлетворяет условию теоремы 2, ибо она радиальна $[9$, c. 120$]$ и $\int_{P} f(x) d x=\int_{H} f(x) d x$.

При $r=1$ гладкие ненулевые радиальные функции с нулевым интегралом по $B_{1}$ удовлетворяют условию теоремы 2 .

Донецкий государственный университет Поступило 01.10 .93

\section{СПИСОК ЦИТИРОВАННОЙ ЛИТЕРАТУРЫ}

[1] Zalcman L. A bibliographical survey of the Pompeiu problem. Approximation by solution of partial differential equations, quadrature formula and related topics: Kluwer Academic Publishers, 1992.

[2] Berenstein C. A., Gay R. Le probleme de Pompeiu locale // J. Analyse Math. 1989. V. 52. P. 133-166.

[3] Berenstein C. A., Gay R. A local version of the two-circles theorem // Israel J. Math. 1986. V. 55. P. 267-288.

[4] Волчков В. В. О функциях с нулевыми интегралами по кубам // Укр. матем. ж. 1991. T. 43. № 6. С. 859-863.

[5] Szabo G. On fuctions having the same integral on congruent semidiscs // Ann. Univ. Sci. Budapest. Lec. Computator. 1982. V. 3. P. 3-9.

[6] Виленкин Н.Я. Специальные функции и теория представлений групп. М.: Наука, 1991.

[7] Волчков В. В. Теоремы о взвешенных сферических средних для некоторых полиномов. Деп. УкрИНТЭИ. № 345-Ук93. Донецк: ДонГУ, 1993.

[8] Стейн И., Вейс Г. Введение в гармонический анализ на евклидовых пространствах. М.: Мир, 1974.

[9] Хелгасон С. Группы и геометрический анализ. М.: Мир, 1987.

[10] Волчков В. В. Теоремы единственности для кратных лакунарных тригонометрических рядов // Матем. заметки. 1992. Т. 51. №6. С. 27-31.

[11] Левин Б. Я. Распределение корней целых функций. М.: ГИТТЛ, 1956. 